\title{
Inflammation-related alterations of lipids after spinal cord injury revealed by Raman spectroscopy
}

Sandra Tamosaityte

Roberta Galli

Ortrud Uckermann

Kerim H. Sitoci-Ficici

Maria Koch

Robert Later

Gabriele Schackert

Edmund Koch

Gerald Steiner

Matthias Kirsch 


\title{
Inflammation-related alterations of lipids after spinal cord injury revealed by Raman spectroscopy
}

\author{
Sandra Tamosaityte, ${ }^{\mathrm{a}, \dagger}$ Roberta Galli, ${ }^{\mathrm{a}, *, \dagger}$ Ortrud Uckermann, ${ }^{\mathrm{b}}$ Kerim H. Sitoci-Ficici, ${ }^{\mathrm{b}}$ Maria Koch, ${ }^{\mathrm{b}, \mathrm{c}}$ \\ Robert Later, ${ }^{\mathrm{b}}$ Gabriele Schackert, ${ }^{\mathrm{b}}$ Edmund Koch, ${ }^{\mathrm{a}}$ Gerald Steiner, ${ }^{\mathrm{a}}$ and Matthias Kirsch $^{\mathrm{b}, \mathrm{c}}$ \\ ${ }^{a}$ TU Dresden, Klinisches Sensoring und Monitoring-Klinik für Anästhesiologie und Intensivtherapie, Fetscherstr. 74, 01307 Dresden, Germany \\ ${ }^{\text {b}}$ TU Dresden, Neurochirurgie, Universitätsklinikum Carl Gustav Carus, Fetscherstr. 74, 01307 Dresden, Germany \\ ${ }^{\circ}$ CRTD/DFG-Center for Regenerative Therapies Dresden-Cluster of Excellence, Fetscherstr. 105, 01307 Dresden, Germany
}

\begin{abstract}
Spinal cord injury (SCl) triggers several lipid alterations in nervous tissue. It is characterized by extensive demyelination and the inflammatory response leads to accumulation of activated microglia/macrophages, which often transform into foam cells by accumulation of lipid droplets after engulfment of the damaged myelin sheaths. Using an experimental rat model, Raman microspectroscopy was applied to retrieve the modifications of the lipid distribution following SCl. Coherent anti-Stokes Raman scattering (CARS) and endogenous two-photon fluorescence (TPEF) microscopies were used for the detection of lipid-laden inflammatory cells. The Raman mapping of $\mathrm{CH}_{2}$ deformation mode intensity at $1440 \mathrm{~cm}^{-1}$ retrieved the lipid-depleted injury core. Preserved white matter and inflammatory regions with myelin fragmentation and foam cells were localized by specifically addressing the distribution of esterified lipids, i.e., by mapping the intensity of the carbonyl Raman band at $1743 \mathrm{~cm}^{-1}$, and were in agreement with CARS/TPEF microscopy. Principal component analysis revealed that the inflammatory regions are notably rich in saturated fatty acids. Therefore, Raman spectroscopy enabled to specifically detect inflammation after SCI and myelin degradation products. @ 2016 Society of Photo-Optical Instrumentation Engineers (SPIE) [DOI: 10.1117/1.JBO.21.6.061008]
\end{abstract}

Keywords: Raman spectroscopy; multiphoton processes; tissues; biomedical optics.

Paper 150684SSR received Oct. 15, 2015; accepted for publication Apr. 11, 2016; published online May 4, 2016.

\section{Introduction}

The spinal cord is part of the central nervous system (CNS). It is formed by white matter located strictly aligned long nerve fibers, and by gray matter, which contains the neuronal cell bodies. The nerve fibers, named axons, are covered by sheaths of myelin, an insulating lipid-based substance responsible for the saltatory action potential propagation. Several types of glial cells perform essential functions both in white and gray matter and influence the neuronal function: astrocytes regulate homeostasis and interaction between synapses, oligodendrocytes form the myelin sheaths, microglia are the resident immune cells within neuronal tissue. ${ }^{1}$

Spinal cord injury (SCI) is the result of an initial physical trauma followed by degenerative processes. Necrotic cell death and hemorrhage take place immediately after induction of the lesion. Secondary events including inflammation, edema, and ischemia lead to additional cell death and axonal degeneration, culminating in the formation of a scar. ${ }^{2}$ The pathological changes in the white matter of spinal cord after injury involve gradual thinning and degradation of the myelin sheath. ${ }^{3}$ The axonal damage and the demyelination that follow the injury lead to permanent functional impairment. ${ }^{4}$

The clearance of myelin fragments after injury involves activated microglia and macrophages. Microglia rapidly responds to various kinds of CNS injury by transforming into large phagocytes, thereby removing debris. Additionally, invading macrophages migrate from the blood in the injured region after

*Address all correspondence to: Roberta Galli, E-mail: Roberta.Galli@ tu-dresden.de

†These authors contributed equally. blood brain barrier damage and contribute to myelin clearance. ${ }^{5}$ Myelin has very high lipid content (70\% to $75 \%$ of its dry weight)..$^{5}$ Demyelinated lesions are thus characterized by the presence of lipid-laden macrophages, which develop the distinctive morphology of foam cells by engulfment of large amounts of myelin-derived lipids. ${ }^{6}$ Foam cells originate both from activated microglia and infiltrating macrophages. ${ }^{7}$

It is well known that cytoplasmatic lipid droplets in foam cells mainly consist of lipid esters covered by a phospholipid monolayer. The lipid metabolism within the macrophages is complex and was investigated in several studies, partly leading to contradictory results. ${ }^{8,9}$ Macrophages in SCI contain myelin, as demonstrated by analysis of histological staining. ${ }^{10,11}$ This was also confirmed by in vitro studies: macrophages that were incubated with myelin transformed into foam cells mainly laden with intracellular droplets of myelin. However, the same study revealed the presence of neutral lipids, too. ${ }^{6}$ It has been widely demonstrated that the composition of lipid droplets in foam cells is very sensitive to the types of lipids that were engulfed by the cell. Macrophages that were cultured in vitro accumulated both saturated and unsaturated fatty acids, ${ }^{12}$ as well as triglycerides ${ }^{13}$ and cholesteryl fatty acid esters, ${ }^{14}$ depending on the composition of the culture media. Microglia activation in vitro leads to the accumulation of lipid droplets and biosynthesis of triglyceride as well. ${ }^{15}$

Standard techniques for studying SCI-induced tissue alterations are based on the use of histological and immunohistochemical methods, which require extended tissue processing and cannot be applied in vivo. Chemical fixatives do not react with most lipids, so lipid bodies are not preserved in 
conventional morphologic methods and are vacant in histological sections. ${ }^{8}$ The use of noninvasive label-free techniques that can be applied on the fresh, unfixed tissue is thus beneficial for study of lipids in SCI and becomes mandatory for in vivo monitoring. Coherent anti-Stokes Raman scattering (CARS) microscopy is fast, intrinsically confocal, and effective in imaging of myelin and lipid bodies on native tissue. ${ }^{16,17}$ Raman spectroscopy is an optical technique that can be applied both on fresh, unfixed tissue and in vivo to retrieve the entire biochemical information.

The overlap of the characteristic Raman signals from the different tissue biomolecules, such as proteins, lipids, nucleic acids, and carbohydrates, encodes a large amount of information potentially useful for diagnostic purposes ${ }^{18}$ and the combination of spectroscopy and microscopy enables the localized biochemical characterization of tissue.

Despite their potential, Raman spectroscopy was rarely applied to investigate SCI. Saxena et al. ${ }^{19}$ applied point Raman measurements to study demyelination and chondroitin sulfate proteoglycan upregulation after spinal cord hemisection. In a previous study, we applied infrared and Raman microspectroscopy in combination with multiphoton microscopy for the detection of lipid depletion and fibrotic scarring after SCI in a rat model of hemimyelonectomy, ${ }^{20}$ as well as for assessing the effects of alginate implants on scarring and demyelination. ${ }^{21}$

Here, we focused on inflammation and retrieved the biochemical information about lipid alterations following SCI in rat models by application of Raman microspectroscopy. CARS microscopy was used to differentiate myelin sheaths, myelin debris, and lipid droplets. Endogenous two-photon fluorescence (TPEF) was used to localize inflammatory cells. ${ }^{22}$

\section{Methods}

\subsection{Animal Experiments and Sample Preparation}

A 2-mm long hemisection of spinal cords at the level T9 of thoracic vertebrae was surgically induced in adult Wistar female rats as described elsewhere. ${ }^{20,21}$ Five animals were included in the study (indicated as \#1 to \#5); one animal received an unfunctionalized alginate hydrogel implant in the lesion (\#5). After 1 month, the animals were perfusion-fixed using $4 \%$ paraformaldehyde (PFA) in phosphate-buffered saline. The spinal cord was removed and postfixed in PFA for $24 \mathrm{~h}$ at $4^{\circ} \mathrm{C}$. Cryoprotection in rising sucrose concentration $(10 \%$ for $24 \mathrm{~h}$ and $30 \%$ for $24 \mathrm{~h}$ ) was followed by embedding the isolated spinal cord in tissue freezing medium (Leica, Nussloch, Germany). The samples were snap frozen on dry ice and stored at $-80^{\circ} \mathrm{C}$. Longitudinal cryosections of $16 \mu \mathrm{m}$ thickness were prepared on $\mathrm{CaF}_{2}$ slides for Raman spectroscopy. Consecutive sections were prepared on glass slides for CARS/TPEF microscopy and histology. The sections were stored at $-20^{\circ} \mathrm{C}$ until use and allowed to thaw for $30 \mathrm{~min}$ prior to spectroscopic analysis or further histological processing.

All animal experiments were performed in accordance with the guidelines of the Dresden University of Technology and were approved by the Regierungspräsidium Dresden, Germany (AZ 24-9168.11-1/2013-37).

\subsection{Hematoxylin and Eosin Staining}

The sections were fixed in methanol-acetone $(1: 1)$, washed in aqua dest, and incubated in Meyer's hematoxylin/hemalum for
3 min. After washing in aqua dest, the tissue was briefly destained in $\mathrm{HCl}-$ ethanol solution. Washing using tap water for $5 \mathrm{~min}$ was followed by $3 \mathrm{~min}$ staining in eosin (1\% eosin $\mathrm{G}$ in $80 \%$ ethanol). The sections were dehydrated with rising ethanol concentrations, cleared in xylene and coverslipped using DePex.

\subsection{Raman Spectroscopy}

Raman spectroscopy was performed as described elsewhere. ${ }^{20}$ Briefly, the excitation was performed with a 785-nm diode laser, propagated with a $100-\mu \mathrm{m}$ optical fiber, and focused by a $50 \times / 0.75$ microscope objective, leading to a focal spot of about $20 \mu \mathrm{m}$. The Raman scattering was collected in reflection configuration and sent to the spectrograph (Raman Rxn1, Kaiser Optical Systems Inc., Ann Arbor, Michigan). Raman spectra were acquired in the range 150 to $3250 \mathrm{~cm}^{-1}$. The spectral resolution was $4 \mathrm{~cm}^{-1}$. An integration time of $750 \mathrm{~ms}$ and two accumulations were used for spectra acquisition. Maps were recorded with a step size of $23 \mu \mathrm{m}$ both in $x$ and $y$ directions.

The datasets were imported in MATLAB (MathWorks Inc., Natick, Massachusetts). The spectra were reduced to the region 450 to $1800 \mathrm{~cm}^{-1}$. A variable baseline was calculated for each spectrum (function "msbackadj" of the MATLAB Bioinformatics Toolbox) and normalization was obtained by standardizing the area under the spectra (function "msnorm" of the MATLAB Bioinformatics Toolbox).

Maps were generated by plotting the intensity of selected bands. The band intensities were calculated as area under the curves in the following range: 1375 to $1455 \mathrm{~cm}^{-1}$ (for evaluation of lipids) and 1728 to $1758 \mathrm{~cm}^{-1}$ (for evaluation of lipid esterification). Principal component analysis (PCA) was performed on a dataset formed by the spectral data of all samples. The function "pca" of the Statistics and Machine Learning Toolbox of MATLAB was used. The score values were reassembled and used to plot the intensity maps of each sample.

\subsection{Coherent Anti-Stokes Raman Scattering/ Endogenous Two-Photon Fluorescence Microscopy}

CARS imaging was performed as described elsewhere. ${ }^{20}$ Shortly, 2-ps Erbium fiber lasers emitting at 780 and 1005 nm (Femto Fiber Pro NIR and TNIR, Toptica, Gräfelfing, Germany) were used to excite the symmetric stretching vibration of methylene at $2850 \mathrm{~cm}^{-1}$. A laser scanning microscope (AxioExaminer with LSM7, Carl Zeiss Microscopy GmbH, Jena, Germany) was used to raster the laser beams, which were focalized on the samples by a $20 \times / 1.0$ water immersion objective. Using nondescanned detection, the CARS signal was collected in transmission (band-pass filter 633 to $647 \mathrm{~nm}$ ). The endogenous TPEF simultaneously generated together with CARS by the ultrashort laser excitation was collected in reflection configuration in the range 500 to $550 \mathrm{~nm}$. CARS and TPEF images were merged using complementary colors (CARS: magenta, TPEF: green) to comply with color perception deficiencies. ${ }^{23}$

\section{Results and Discussion}

Five samples of SCI showing different degrees of inflammatory responses were selected according to the hematoxylin and eosin (H\&E) staining and are shown in Figs. 1(a)-1(e). H\&E is one of the primary stains used in histology and is the gold standard for 


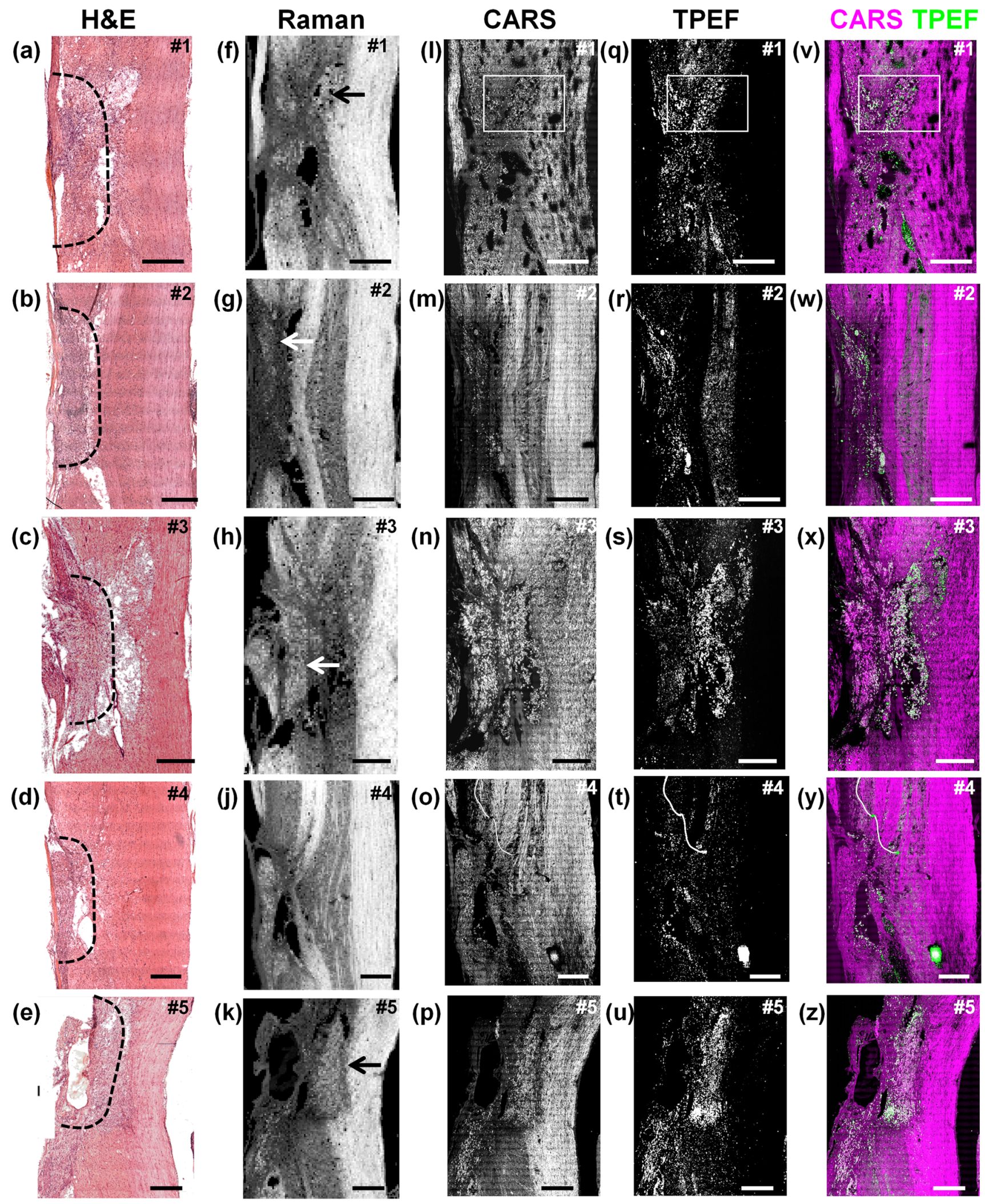

Fig. 1 Cryosections of $\mathrm{SCl}$ in rat models. (a-e) H\&E staining; (f-k) Raman intensity maps of the band at $1440 \mathrm{~cm}^{-1}\left(\mathrm{CH}_{2}\right.$ deformation vibration); (I-p) CARS image (tuned to excite the $\mathrm{CH}_{2}$ symmetric stretching vibration); (q-u) endogenous TPEF; ( $\mathrm{v}-\mathrm{z}$ ) merged CARS (magenta) and TPEF (green) images. Scale bar: $0.5 \mathrm{~mm}$. The dotted lines in $(\mathrm{a}-\mathrm{e})$ indicate hemisection positions. Arrows in $(\mathrm{f}-\mathrm{h})$ and $(\mathrm{k})$ indicate lipid-rich areas in the injured region. The boxes in (I), (q), and (v) indicate the position of zoom-in images of Fig. 2. 
pathology. Hematoxylin is a blue stain that binds to nucleic acids. Eosin is a pink stain that binds to acidophilic substances such as proteins. The samples in Figs. 1(a), 1(c), and 1(e) display extended inflammation inside and around the lesion core; the sample in Fig. 1(b) has areas of inflammation at the lesion site only, while the sample in Fig. 1(d) has less inflammatory cells that are mainly localized near the large cysts. Cysts are located mainly at the lesion site, which is indicated by the dotted line in the figure. Raman maps of several square millimeters in size covering the lesion site and the surrounding regions were acquired and used to visualize the distribution of lipid species inside the nervous tissue [Figs. 1(f)-1(k)] by plotting the intensity of the band at $1440 \mathrm{~cm}^{-1}$, which is attributed to deformation vibrations of $\mathrm{CH}_{2}$ functional groups. ${ }^{24}$ The lesion core is generally poorer of lipids when compared to the surrounding tissue and therefore can be readily recognized. This indicates the lack of nervous tissue at the injury site and subsequent demyelination in adjacent regions, in agreement with previous results. ${ }^{20}$ Areas with high lipid content are visible inside or immediately nearby the lesion in four samples [see arrows in Figs. 1(f)-1(h) and $1(\mathrm{k})$ ]. However, the Raman maps did not enable single axons to be recognized (at least with the lateral resolution that characterizes our system) and therefore did not show whether the high lipid content is due to the presence of functional myelinated axons.

CARS microscopy addressing $\mathrm{CH}_{2}$ symmetric stretching vibration was applied in order to combine the chemical information about lipid distribution with the cellular morphology of the tissue, and therefore to identify axons and cells. The amount of biochemical information is traded-off at the advantage of speed, and label-free imaging of whole sections is performed in minutes. The combination of the morphochemical information supplied by CARS microscopy with the biochemical fingerprint of lipid species provided by Raman spectroscopy was already assessed for investigation of lipid droplets. ${ }^{25}$

On sections of spinal cord tissue, CARS microscopy provides the overall distribution of lipids, thereby visualizing demyelination at the lesion site in a way similar to Raman mapping based on intensity of a $\mathrm{CH}_{2}$ deformation band [compare Raman maps in Figs. 1(f)-1(k)] and CARS images in Figs. 1(1)1(p). Due to the submicrometric lateral resolution and the inherent confocality, CARS microscopy allows the visualization of subcellular structures. Figure 2 shows in more detail the region with high lipid content immediately nearby the lesion of sample \#1 and illustrates the tissue micromorphology. The H\&E staining in Fig. 2(a) shows the microcystic morphology of damaged nervous tissue (on the left side of images), compared to the compact and highly ordered white matter in the contralateral tissue (on the right side of images). The CARS image in Fig. 2(b) provides additional morphochemical information: lipid droplets and fragmented myelin sheaths are localized in the damaged tissue, while the contralateral white matter is characterized by a strictly aligned axonal morphology. The simultaneous acquisition of TPEF [Fig. 2(c)] adds information about the extent of microglia/macrophages activation and therefore localizes the inflammation. $^{20,22}$ TPEF shows a clear overlap with the distribution of lipid droplets in the inflammatory region, while no fluorescent cells are present in the normal white matter [merged CARS and TPEF images in Fig. 2(d)]. Therefore, it constitutes an indicator for the presence of foam cells, i.e., activated microglia/macrophages that got laden with cytoplasmatic lipid droplets after engulfment of large quantities of myelin debris. The comparison with the H\&E staining confirms the presence of foamy macrophages in the same regions.

The high lipid content regions indicated by arrows in the Raman maps of Figs. 1(f)-1(k) correspond to inflammatory regions with disrupted myelin sheaths, presence of fluorescent foam cells and devoid of integer myelinated axons, as identified by CARS-TPEF imaging. This clearly shows that the lipid distribution by spectroscopic mapping of the deformation band of $\mathrm{CH}_{2}$ groups is not exhaustive for evaluation of white matter injury. In the CNS, myelin clearance is slow: ${ }^{26,27}$ as myelin debris and foam cells remain in the tissue for weeks after the injury, the sole evaluation of the lipid content does not allow

\section{(a)}
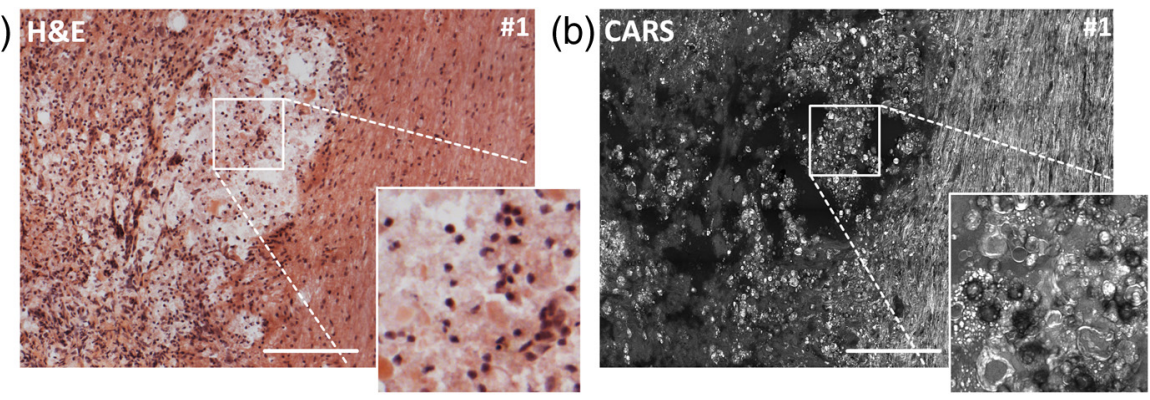

(c)
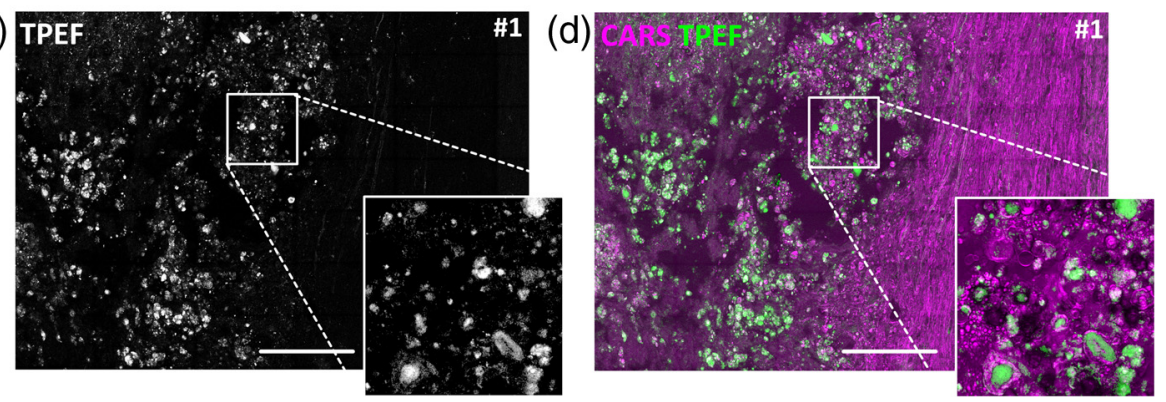

Fig. 2 Region with high lipid content in more detail. (a) H\&E staining, (b) CARS, (c) TPEF, and (d) merged
CARS/TPEF images comparing morphology of normal white matter in preserved spinal cord and foam

Fig. 2 Region with high lipid content in more detail. (a) H\&E staining, (b) CARS, (c) TPEF, and (d) merged
CARS/TPEF images comparing morphology of normal white matter in preserved spinal cord and foam cells in the injured rat spinal cord (sample \#1). Scale bar: $200 \mu \mathrm{m}$. 
distinguishing between preserved regions of white matter with properly myelinated axons and regions of ongoing fragmentation of myelin sheaths and inflammation.

After SCI, myelin debris is taken up by phagocytic cells. Myelin is composed by cholesterol, phospholipids, galactolipids, and plasmalogens in a molar ratio of approximately 2:2:1:1. ${ }^{5}$ Therefore, the metabolites of cholesterol and phospholipids are expected to determine the composition of lipid droplets in macrophages and their spectral markers were analyzed to retrieve the inflammation.

Intracellular lipid droplets are dynamic organelles, ${ }^{28}$ where cholesterol is stored as cholesteryl esters. The sterol ring vibrations in free cholesterol and cholesteryl esters produce a sharp and characteristic Raman band at $697 \mathrm{~cm}^{-1} \cdot{ }^{29,24}$ However, a mapping of the sterol vibration band intensity does not enable to visualize the inflammation (data not shown). The esterification of cholesterol is opposed by neutral cholesteryl esters hydrolysis, which is critical for maintenance of cellular cholesterol homeostasis. ${ }^{30}$ The free cholesterol is either released by macrophages in the intracellular space to be recycled on site, or is removed by migration of macrophages away from the injury, but no degradation of the sterol ring takes place in foam cells, since mammalian cells do not have this ability. ${ }^{30}$ This explains why monitoring the sterol ring distribution by Raman spectroscopy is not useful for detection of degrading myelin and foam cells.

Lipid esterification is the key process for storage in lipid droplets. Also phospholipids are completely hydrolyzed in foam cells to produce a pool of free fatty acids ${ }^{31}$ that are subsequently esterified. ${ }^{32}$ The band of carbonyl group stretching vibration at $1743 \mathrm{~cm}^{-1} 33,34$ constitutes a marker of cholesteryl and fatty acid esters in Raman spectra. The carbonyl vibration intensity maps of all samples are shown in Fig. 3. The highest degree of lipid esterification is located in the injured regions and colocalizes with the foam cells as detected with CARS/TPEF microscopy (compare with Fig. 1). The average Raman spectrum calculated in an injured tissue region $(12 \times 12$ pixels $)$ characterized by lipid esterification is shown in Fig. 3(f), compared with the average spectrum calculated in a region $(12 \times 12$ pixels $)$ of preserved white matter. The carbonyl band is visible in the spectrum of the injured region and absent in the one of white matter.

For the interpretation of the maps in Fig. 3, it has to be considered that CNS lipids are particularly susceptible to oxidation by virtue of their high content of unsaturated fatty acids. Many studies have demonstrated increased oxidative damage and lipid peroxidation in CNS injuries. ${ }^{35}$ Advanced lipid peroxidation end-products include a variety of reactive carbonyl compounds, ${ }^{36}$ which could contribute to the intensity of band at $1743 \mathrm{~cm}^{-1}$. However, the time frame of lipid oxidation in rat SCI is limited to the acute phase and resolves at around 5 days after injury. ${ }^{37}$ As no significant lipid peroxidation is expected one month postinjury, the maps of Fig. 3 represent the distribution of esterified lipids.

The intensity maps discussed above elucidate the distribution of lipids, sterols, and esterified lipids in the injury region versus normal nervous tissue. However, they do not indicate whether the injured and inflammatory regions are enriched of other types of lipids or fatty acids, which are eventually produced by myelin degradation inside the foam cells or as part of inflammatory pathways. This can be retrieved with multivariate methods, i.e., PCA.

When PCA is performed on the entire dataset (i.e., on the spectral data of all samples merged in one work space), the first to fourth components indicate tissue compounds that are not related to the distribution of inflammation, but represent (a)

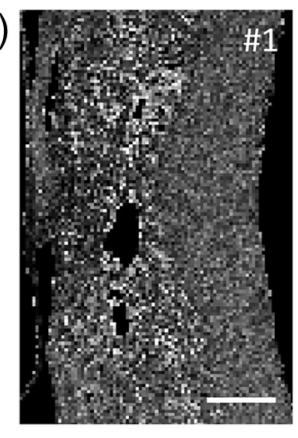

(b)

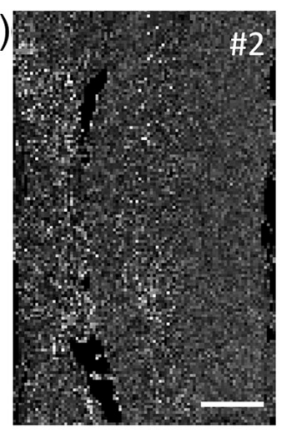

(c)

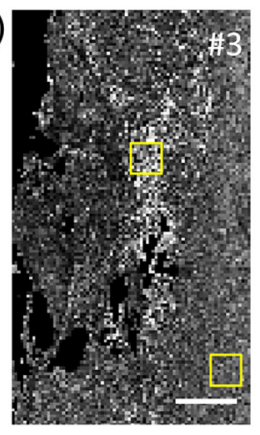

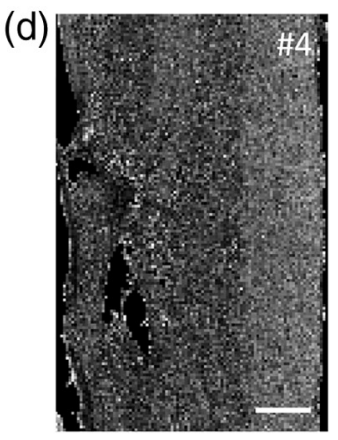

(e)

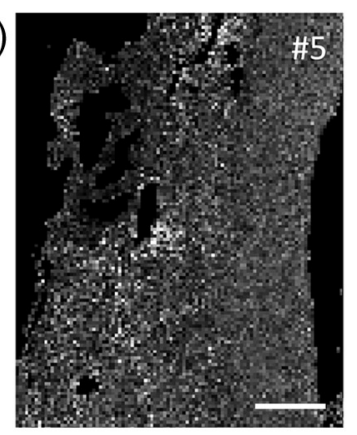

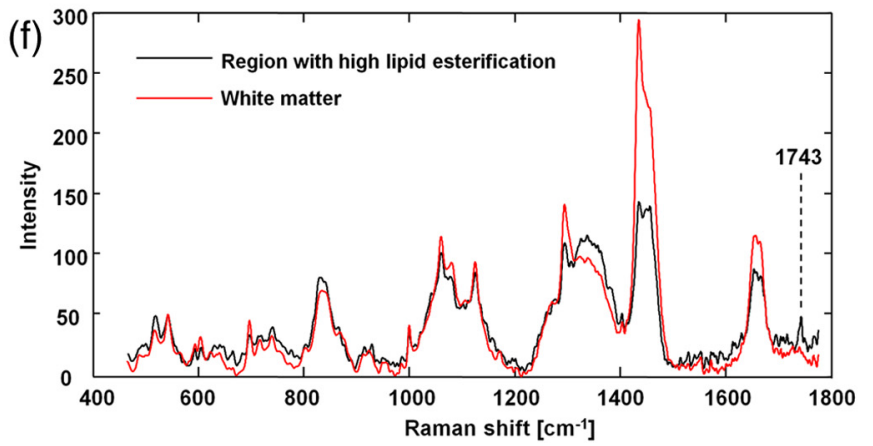

Fig. 3 Carbonyl vibration intensity maps of all samples. (a)-(e) Maps of intensity of the carbonyl stretching vibration at $1743 \mathrm{~cm}^{-1}$, illustrating the distribution of esterified lipids. Scale bar: $0.5 \mathrm{~mm}$. (f) Raman spectra of regions with high lipid esterification (injury) and low lipid esterification (white matter); the spectra were calculated by average in regions $12 \times 12$ pixels large, as indicated by boxes in (c). 
other tissue constituents (as for example lipids and collagen, data not shown). The fifth component clearly appears to be associated to the presence of activated microglia/macrophages (Fig. 4). As it can be seen by comparing the maps of Figs. 4(a)4(e) with the TPEF images of Figs. 1(q)-1(u), high pixel intensity areas-representing higher score-identify the inflammatory regions with accumulation of fluorescent macrophages. The colocalization with lipid droplets in the CARS image and with TPEF is shown with more detail in Figs. 4(g)-4(i). The fraction of variance associated to this component is as small as $0.13 \%$, indicating a compound with very low concentration within the tissue. The corresponding loading vector [Fig. 4(f)] is consistent with saturated fatty acids. The positive narrow signals at 1060 and $1126 \mathrm{~cm}^{-1}$ are attributed to $\mathrm{C}-\mathrm{C}$ stretching vibration, and the ones at 1293 and $1434 \mathrm{~cm}^{-1}$ are attributed to $\mathrm{CH}_{2}$ and $\mathrm{CH}_{3}$ deformation vibration. ${ }^{34,38}$ The negative spectral contribution at $1658 \mathrm{~cm}^{-1}$-attributed to $\mathrm{C}=\mathrm{C}$ stretching vibration ${ }^{34}$ - confirms that this component is related to a saturated type of lipid.

It was recently reported that the acute activation of microglial cells results in increased content of cellular total saturated fatty acids, especially of palmitic acid (C16:0) and stearic acid (C18:0). ${ }^{39}$ However, a precise evaluation of the spectral pattern of the loading vector strongly suggests the presence of myristic acid (C14:0), matching with high precision the band positions as reported in Ref. 34. For instance, the four most intense bands of myristic acids are reported at 1433, 1294, 1125, and $1062 \mathrm{~cm}^{-1}$, while the same bands of both palmitic and stearic acids are all shifted 5 to $12 \mathrm{~cm}^{-1}$ toward the higher wavenumber. As no changes of myristic acid content in microglia are reported upon activation, ${ }^{39}$ an increase in myristic acid might be explained by the increased density of inflammatory cells compared to other tissue regions.

As the lateral resolution of Raman spectroscopy in our experiments is not able to identify the lipid droplets from other cellular compartments, it is unclear whether the saturated fatty acids are a product of myelin degradation that are stored in the lipid droplets. Only the upregulation of saturated fatty acids in activated microglia was addressed in Ref. 39; their subcellular localization and a possible relationship to the presence of lipid droplets were not studied. Furthermore, no reference reporting an accumulation of saturated fatty acids inside the lipid droplet of immune cells was found, except in cases where they are cultured in media enriched with saturated lipids. The lipids of the CNS contain a very high amount of polyunsaturated fatty acids, particularly of arachidonic acid (C20:4), and docosahexaenoic acid (C22:6). ${ }^{40}$ In fact, necrotic regions with presence of macrophages and foam cells in brain affected by glioblastoma were studied by Raman spectroscopy and the spectral signature of unsaturated fatty acids [i.e., oleic acid (C18:1)] was (a)

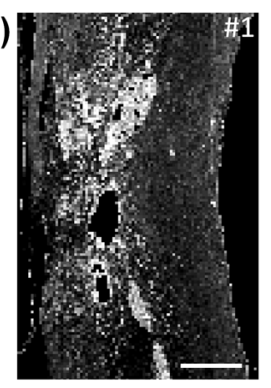

(b)

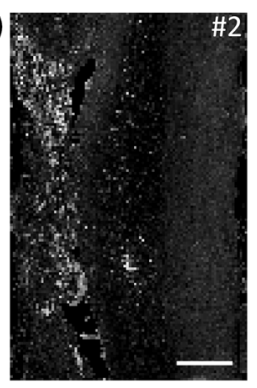

(c)

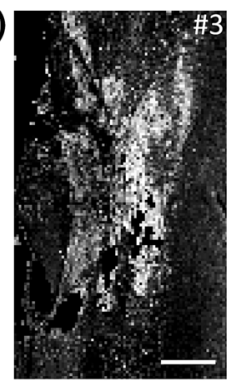

(d)

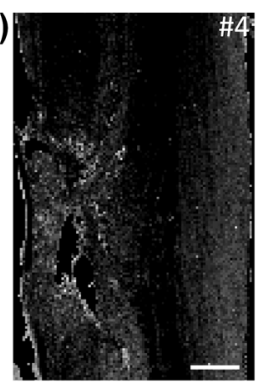

(e)
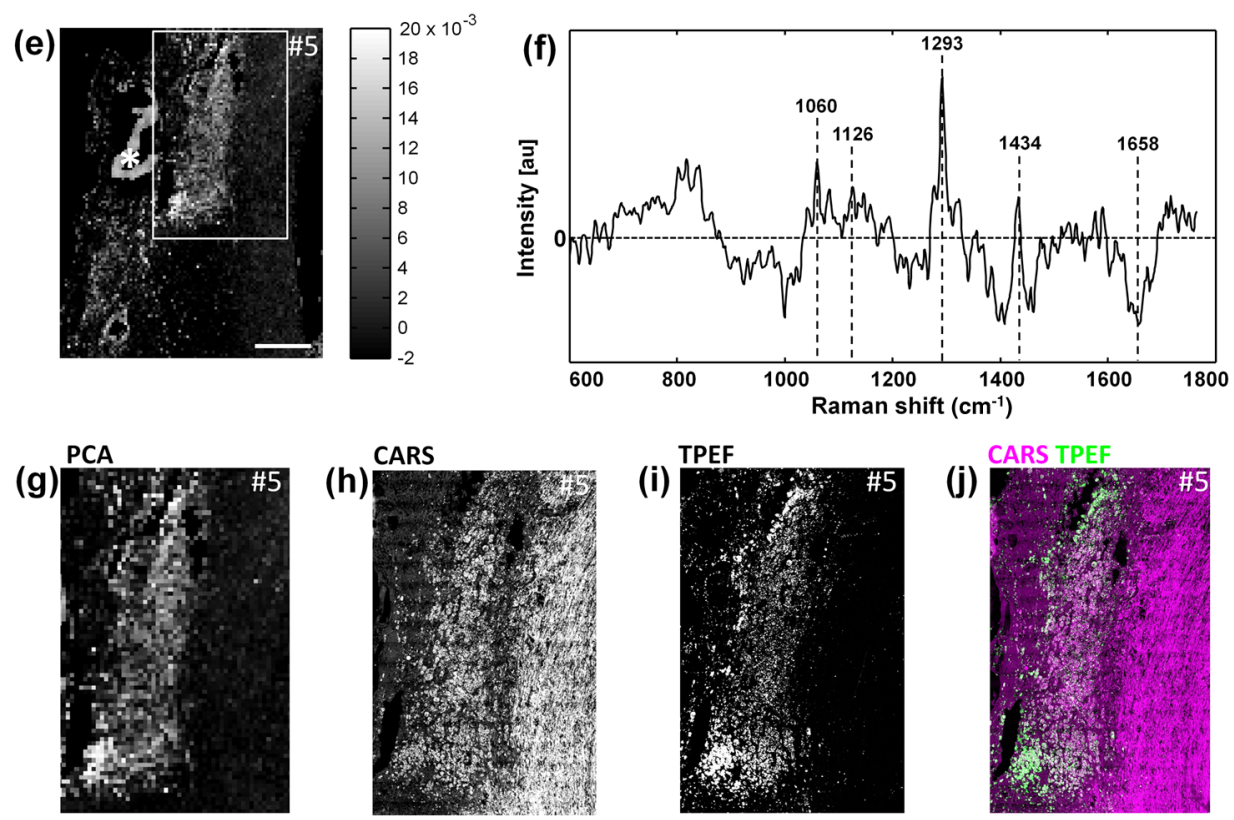

Fig. 4 PCA: (a-e) maps of score values for all samples and (f) loading vector for the fifth component. (g) Zoom-in of the box in the score map of sample \#5 shown in (e); (h-j) CARS, TPEF, and merged images of sample \#5 in the same region shown in (g). Scale bar: $0.5 \mathrm{~mm} .{ }^{*}$ in $(\mathrm{e})$ indicates the alginate implant. 
detected. ${ }^{41}$ Studies made by Raman microspectroscopy on macrophages in vitro indicated esterified unsaturated lipids as a main component of the lipid droplets. ${ }^{42,43}$ Lipid droplets in leucocytes were found to be rich in unsaturated moieties by resonance Raman spectroscopy and provide a reservoir of arachidonic acid, which is involved in the activation pathway of immune cells. ${ }^{44}$ Therefore, the available bibliography does not provide sufficient information to relate the overexpression of saturated fatty acids inside the injured spinal cord with the storage of myelin degradation products inside the lipid droplets of foam cells. However, the comparison with CARS and TPEF images provides further indication.

By inspecting in detail the spatial distribution of signals in the inflammatory regions, it is possible to clearly see that the score maps in fact mimic the pattern of TPEF more than the distribution of lipid droplets as seen by CARS [compare Figs. 4(g) with 4(h) and 4(i)]. This suggests a correlation with the activation state of the immune cells. It was hypothesized that palmitic and stearic acids may play a role in the activation of a pathway that modulates the expression of inflammatory cytokines. ${ }^{39}$ It is also known that myristic acid has an important role in the cell lipid metabolism, by regulating the production of polyunsaturated fatty acid (e.g., of docosahexaenoic acid). ${ }^{45}$ Moreover, recent findings suggest that myristic acid may have a specific regulatory role because of its ability to modify enzyme activities or protein functions through their $\mathrm{N}$-terminal myristoylation. In particular, myristic acid regulates the activity of mammalian desaturases, which are enzymes involved in the metabolism of lipids. ${ }^{46}$ Therefore, it is more likely that the small amounts of saturated fatty acids identified by PCA in foam cell-rich regions of spinal cord are not just stored in the lipid droplets, but play an active role in the metabolism of activated microglia/macrophages.

\section{Conclusions}

SCI triggers degenerative processes characterized by scarring, demyelination, and inflammation. While demyelination and scarring were already addressed elsewhere by Raman spectroscopy, ${ }^{20,19}$ inflammation was so far neglected in spectroscopic studies of SCI. Here we showed that: (i) an unspecific mapping of the lipid distribution based on $\mathrm{CH}_{2}$ (and on sterol ring) vibration band intensity does not discern between preserved white matter and inflammatory regions with myelin fragmentation and presence of lipid-laden microglia/macrophages; (ii) inflammatory regions with lipid-laden microglia/macrophages can be localized by addressing the distribution of esterified lipids (i.e., by mapping the intensity of carbonyl Raman band); (iii) these inflammatory regions are enriched with saturated fatty acids, which likely play a role in the lipid metabolism of activated microglia/macrophages. In conclusion, Raman spectroscopy specifically addresses the inflammation taking place in the nervous tissue after SCI, and the products of myelin degradation. Therefore, Raman spectroscopy is confirmed to be a very useful tool for characterization of degenerative events triggered by injury of CNS.

\section{Acknowledgments}

This work was supported by the German Federal Ministry of Education and Research (MediCARS project, A.Z. 13N10777), by the Medical Faculty of the Technische Universität Dresden (Habilitationsförderung für Frauen) and by a seed grant of the CRTD—DFG Research Center for Regenerative Therapies
Dresden, Technische Universität Dresden. The authors gratefully acknowledge the highly professional assistance of Dipl. Biol. Elke Leipnitz and Juliane Hartmann in sample preparation.

\section{References}

1. A. Nógrádi and G. Vrbová, Transplantation of Neural Tissue into the Spinal Cord, 2nd ed., p. 2, Landes Biosciences/Springer, Berlin, Germany (2005).

2. C. Profyris et al., "Degenerative and regenerative mechanisms governing spinal cord injury," Neurobiol. Dis. 15(3), 415-436 (2004).

3. C. J. Ek et al., "Pathological changes in the white matter after spinal contusion injury in the rat," PloS One 7(8), e43484 (2012).

4. A. Alizadeh, S. M. Dyck, and S. Karimi-Abdolrezaee, "Myelin damage and repair in pathologic CNS: challenges and prospects," Front Mol. Neurosci. 8, 35 (2015).

5. K. A. Nave and H. B. Werner, "Myelination of the nervous system: mechanisms and functions," Annu. Rev. Cell Dev. Biol. 30, 503-533 (2014).

6. L. A. Boven et al., "Myelin-laden macrophages are anti-inflammatory, consistent with foam cells in multiple sclerosis," Brain 129, 517-526 (2006).

7. H. Li, M. L. Cuzner, and J. Newcombe, "Microglia-derived macrophages in early multiple sclerosis plaques," Neuropathol. Appl. Neurobiol. 22(3), 207-215 (1996).

8. Y. Ohsaki, M. Suzuki, and T. Fujimoto, "Open questions in lipid droplet biology," Chem. Biol. 21(1), 86-96 (2014).

9. M. Salehipour et al., "Polyunsaturated fatty acids and modulation of cholesterol homeostasis in THP-1 macrophage-derived foam cells," Int. J. Mol. Sci. 11(11), 4660-4672 (2010).

10. M. H. Ma et al., "Monocyte recruitment and myelin removal are delayed following spinal cord injury in mice with CCR2 chemokine receptor deletion," J. Neurosci. Res. 68(6), 691-702 (2002).

11. N. Vallieres et al., "Systemic injections of lipopolysaccharide accelerates myelin phagocytosis during Wallerian degeneration in the injured mouse spinal cord," Glia 53(1), 103-113 (2006).

12. B. X. Wong et al., "Modulation of macrophage fatty acid content and composition by exposure to dyslipidemic serum in vitro," Lipids 46(4), 371-380 (2011).

13. K. R. Feingold et al., "Mechanisms of triglyceride accumulation in activated macrophages," J. Leukoc. Biol. 92(4), 829-839 (2012).

14. I. Tabas, "Cholesterol and phospholipid metabolism in macrophages," BBA-Mol. Cell Biol. L 1529(1-3), 164-174 (2000).

15. A. Khatchadourian et al., "Dynamics and regulation of lipid droplet formation in lipopolysaccharide (LPS)-stimulated microglia," BBA-Mol. Cell Biol. L 1821(4), 607-617 (2012).

16. T. T. Le, S. H. Yue, and J. X. Cheng, "Shedding new light on lipid biology with coherent anti-stokes Raman scattering microscopy," J. Lipid Res. 51(11), 3091-3102 (2010).

17. X. Nan, E. O. Potma, and X. S. Xie, "Nonperturbative chemical imaging of organelle transport in living cells with coherent anti-stokes Raman scattering microscopy," Biophys. J 91(2), 728-735 (2006).

18. D. Naumann, Vibrational Spectroscopy in Microbiology and Medical Diagnostics, pp. 1-8, John Wiley \& Sons, Inc., Hoboken, New Jersey (2008).

19. T. Saxena et al., "Raman spectroscopic investigation of spinal cord injury in a rat model," J. Biomed. Opt. 16(2), 027003 (2011).

20. R. Galli et al., "Vibrational spectroscopic imaging and multiphoton microscopy of spinal cord injury,' Anal. Chem. 84(20), 8707-8714 (2012).

21. R. Galli et al., "Raman-based imaging uncovers the effects of alginate hydrogel implants in spinal cord injury," Proc. SPIE 9536, 95360Y (2015).

22. O. Uckermann et al., "Endogenous two-photon excited fluorescence provides label-free visualization of the inflammatory response in the rodent spinal cord," Biomed. Res. Int. 2015, 859084 (2015).

23. M. Geissbuehler and T. Lasser, "How to display data by color schemes compatible with red-green color perception deficiencies," Opt. Express 21(8), 9862-9874 (2013).

24. Z. Movasaghi, S. Rehman, and I. U. Rehman, "Raman spectroscopy of biological tissues," Appl. Spectrosc. Rev. 42(5), 493-541 (2007). 
25. M. C. Potcoava et al., "Raman and coherent anti-stokes Raman scattering microscopy studies of changes in lipid content and composition in hormone-treated breast and prostate cancer cells," J. Biomed. Opt. 19, 111605 (2014).

26. R. C. Armstrong et al., "White matter involvement after TBI: clues to axon and myelin repair capacity," Exp. Neurol. 275(Part 3), 328-333 (2015).

27. M. E. Vargas and B. A. Barres, "Why is Wallerian degeneration in the CNS so slow?" Annu. Rev. Neurosci. 30, 153-179 (2007).

28. M. S. Brown, Y. K. Ho, and J. L. Goldstein, "The cholesteryl ester cycle in macrophage foam cells. Continual hydrolysis and re-esterification of cytoplasmic cholesteryl esters," J. Biol. Chem. 255(19), 9344-9352 (1980).

29. E. B. Hanlon et al., "Prospects for in vivo Raman spectroscopy," Phys. Med. Biol. 45(2), R1-R59 (2000).

30. M. Sekiya et al., "The role of neutral cholesterol ester hydrolysis in macrophage foam cells," J. Atheroscler. Thromb. 18(5), 359-364 (2011).

31. J. F. Goodrum et al., "Fatty-acids from degenerating myelin lipids are conserved and reutilized for myelin synthesis during regeneration in peripheral-nerve," J. Neurochem. 65(4), 1752-1759 (1995).

32. J. F. Goodrum et al., "Fate of myelin lipids during degeneration and regeneration of peripheral-nerve: an autoradiographic study," $J$. Neurosci. 14(1), 357-367 (1994).

33. J. R. Beattie, S. E. J. Bell, and B. W. Moss, "A critical evaluation of Raman spectroscopy for the analysis of lipids: fatty acid methyl esters," Lipids 39(5), 407-419 (2004).

34. K. Czamara et al., "Raman spectroscopy of lipids: a review," J. Raman Spectrosc. 46(1), 4-20 (2015).

35. R. M. Adibhatla and J. F. Hatcher, "Lipid oxidation and peroxidation in CNS health and disease: from molecular mechanisms to therapeutic opportunities," Antioxid. Redox Signal. 12(1), 125-169 (2010).

36. A. Negre-Salvayre et al., "Advanced lipid peroxidation end products in oxidative damage to proteins. Potential role in diseases and therapeutic prospects for the inhibitors," Br. J. Pharmacol. 153(1), 6-20 (2008).

37. S. D. Christie et al., "Duration of lipid peroxidation after acute spinal cord injury in rats and the effect of methylprednisolone," Neurosurg. Focus 25(5), 1-7 (2008).

38. J. De Gelder et al., "Reference database of Raman spectra of biological molecules," J. Raman Spectrosc. 38(9), 1133-1147 (2007).

39. E. B. Button et al., "Microglial cell activation increases saturated and decreases monounsaturated fatty acid content, but both lipid species are proinflammatory," Lipids 49(4), 305-316 (2014).

40. I. Carrie et al., "Specific phospholipid fatty acid composition of brain regions in mice: effects of $n-3$ polyunsaturated fatty acid deficiency and phospholipid supplementation," J. Lipid Res. 41(3), 465-472 (2000).

41. N. Amharref et al., "Discriminating healthy from tumor and necrosis tissue in rat brain tissue samples by Raman spectral imaging," Biochim. Biophys. Acta-Biomembr. 1768(10), 2605-2615 (2007).

42. C. Matthaus et al., "Noninvasive imaging of intracellular lipid metabolism in macrophages by Raman microscopy in combination with stable isotopic labeling," Anal. Chem. 84(20), 8549-8556 (2012).

43. C. Stiebing et al., "Complexity of fatty acid distribution inside human macrophages on single cell level using Raman micro-spectroscopy," Anal. Bioanal. Chem. 406(27), 7037-7046 (2014).

44. H. J. van Manen et al., "Single-cell Raman and fluorescence microscopy reveal the association of lipid bodies with phagosomes in leukocytes," Proc. Natl. Acad. Sci. United States of America 102(29), 10159-10164 (2005).

45. V. Rioux and P. Legrand, "Saturated fatty acids: simple molecular structures with complex cellular functions," Curr. Opin. Clin. Nutr. Metab. Care 10(6), 752-758 (2007).

46. V. Rioux, F. Pedrono, and P. Legrand, "Regulation of mammalian desaturases by myristic acid: N-terminal myristoylation and other modulations," BBA-Mol. Cell Biol. L 1811(1), 1-8 (2011).

Sandra Tamosaityte received her MS degree in physics from Vilnius University, Vilnius, Lithuania, in 2013, where currently she is pursuing her PhD in physics while doing her research activities at the Faculty of Medicine at the TU Dresden. Her work mainly focuses on application of vibrational spectroscopy and multiphoton microscopy to investigate tissue properties. Currently, she is interested in label-free imaging for monitoring spinal cord injury and regeneration.

Roberta Galli holds a degree in physics from the University of Milan and a PhD in medical technology from the TU Dresden. After many years spent in the R\&D department of a private company, she is now employed at the Faculty of Medicine at the TU Dresden, where she works on optical techniques for label-free tissue characterization. Her main research areas are Raman spectroscopy and multiphoton microscopy.

Ortrud Uckermann is a neurobiologist and works in neurosurgery at the University Hospital at the TU Dresden. Her research areas are nervous tissue pathologies using microscopic and spectroscopic techniques, with focus on biomarkers and translational aspects. She is experienced in animal models, cellular imaging, and physiology based on previous research in neuropediatrics at the TU Dresden and at the Paul-Flechsig Institute of Brain Research at the University of Leipzig.

Kerim H. Sitoci-Ficici graduated from Maximilians University in Munich, where he achieved his academic degree (doctor medicinae). At present, he is a fellow in neurosurgery at the Faculty of Medicine at the TU Dresden. His field of research is spinal cord trauma and axonal regeneration.

Maria Koch holds a BS degree in biotechnology of the University of Applied Sciences Bremen. She spent 2 years working as a research assistant at the National University of Singapore. Currently, she is doing her master's degree at the Center for Regenerative Therapy at the TU Dresden. In the frame of her practical courses she worked at the Faculty of Medicine performing multiphoton microscopy of spinal cord injury.

Robert Later graduated from the Technical University in Dresden in 2011. At present he is a fellow in neurosurgery at the Faculty of Medicine at the TU Dresden. His field of research is spinal cord trauma and visualization of axonal regeneration using label-free optical techniques.

Gabriele Schackert is a chair of the Department of Neurosurgery at the University Hospital at the TU Dresden. She is currently a president of the German Society of Surgery and past president of the German Society of Neurosurgery. Her main research interests are neurooncology, skull base surgery, molecular analysis of brain tumors and cerebral aneurysms, molecular endospectroscopy, and pre- and intraoperative imaging.

Edmund Koch received his diploma and $\mathrm{PhD}$ in physics from the University of Marburg. He worked as a senior researcher at the Drägerwerke AG, Lübeck, and thereafter he became professor for laser and optics at the Lübeck University of Applied Sciences. He is currently head of the work group Clinical Sensoring and Monitoring at the Faculty of Medicine at the TU Dresden. His main research interests are optical coherence tomography and spectroscopic imaging.

Gerald Steiner is docent for analytical chemistry and group leader of Spectral Diagnostic Research at the TU Dresden, Faculty of Medicine. He worked for many years in analytical chemistry. His general research interests lie in the field of optical spectroscopy and biosensing. Currently his research is focused on in situ biospectroscopy including new imaging modalities and label-free detection of functional and pathological biological matter.

Matthias Kirsch is a neurosurgeon at the University Hospital at the TU Dresden and group leader of Experimental Neuro-oncology and Translational Neurosurgical Research. His research focuses on brain tumors and regeneration, including experimental therapies, optical spectroscopic analyses and intraoperative integration of multimodal imaging. 